\title{
Properties of solids under high pressure-An electronic band structure approach
}

\author{
R ASOKAMANI and C RAVI \\ Department of Physics, Anna University, Chennai 600 025, India
}

\begin{abstract}
The predicting capability of various parameters related to solids by performing their high pressure electronic band structures is discussed in detail. The studies on ground state crystal structure, magnetic structure and magnetic phase transitions under pressures, $s-d$ electron transitions, bulk modulus, Debye temperature, elastic constants, insulator to metal transitions and the phenomenon of pressure induced superconductivity will be discussed with examples. Especially the method of calculating the Debye temperature for a large number of ternary chalcopyrite systems using their recently evahuated band structure will be presented. The limitations of the band theory when used with the local density approximation with respect to the determination of band gaps and magnetic properties will also be discussed.
\end{abstract}

Keywords. Solids; electronic band approach.

\section{Introduction}

The evolution of band structure methods have led to considerable amount of understanding of the various properties of materials in the past few decades. The augmented plane wave (APW) method which was proposed by Slater (1937) was one of the widely used methods to calculate the band structure employing the Slater exchange for the contribution of the muffin tin potential. The band structure calculations yield the Fermi energy $\left(E_{\mathrm{F}}\right)$ and the density of states at $E_{\mathrm{F}}, N\left(E_{\mathrm{F}}\right)$ using which the electronic specific heat coefficient was calculated. Usually this value was compared with the experjmental value to check the validity of the method. One of the major steps in the application of band structure to study the mechanical property such as the bulk modulus was performed by Moruzzi et al (1978) for several elemental solids using the KKR method. These calculations were extended by Papaconstantopolous (1986) for evaluating their superconducting transition temperatures. Papaconstantopolous et al (1977) have also used the APW method in the systematic study of the band structure of a number of elemental solids which also included the spin polarized calculations of some of the ferromagnetic metals.

The introduction of linear methods by Andersen (1975) is a major breakthrough in the calculations of band structures as it vastly reduces the computational time and they are also self consistent although self consistency can be achieved by other methods. Skriver $(1984,1985)$ adopted the linear muffin tin orbital (LMTO) method for calculating the total energy for different metals in the periodic table and it has improved our knowledge with regard to the correlation between the $d$ electron number and crystal structural stability. The total energy calculations were performed in a similar manner using pseudopotential method by Cohen for some of the semiconductors. Thus the band structure calculations even performed under ambient conditions can be used to calculate quantities such as the electronic specific heat coefficient $(\chi)$, the Pauli spin susceptibility $\left(\chi_{p}\right)$ and the electronic charge density distribution as well as their momentum distribution in solids (Mijnarends 1973).

In 70's with the advent of high pressure diamond anvil devices Wittig and Probst $\left({ }^{\prime} 78\right)$ studied the phenomenon of pressure induced st srconductivity in several metals. Experimental observations with regard to crystal structure modifications, the phenomenon of insulator-metal transition, superconductivity as well as magnetic phase changes at high pressures have been made for a large number of systems. The band theory has reached such a high level of sophistication so as to enable the study of all the above mentioned phenomena. In this paper we shall discuss certain case studies which are of recent origin.

\section{Superconductivity in ionic solids}

One of the most exciting experimental observations which has been currently reported is the superconductivity in an ionic solid such as CsI (figure 1) and the same was predicted by us from band structure calculations as early as 1991 (Asokamani et al 1991; Eremets et al 1998). The result is of fundamental importance because of the fact that till date the only other ionic solid in which superconductivity is said to have been observed is $\mathrm{NaCl}$. Pressure induced superconductivity is very well known in metals, semiconductors and van der Waals solids (figure 2). The existence of certain systematics lead to 
the question as to 'why superconductivity was not observed in ionic solids?' and this has been raised earlier (Asokamani et al 1997). Pressure induced superconductivity in group III transition metals Sc, Y and La and as well as in other groups show the general trend viz that the pressure required to drive a material to go to the superconducting state $\left(P_{\mathrm{s}}\right)$ depends upon the radius of the atom. As the atomic radius decreases the pressure required $P_{\mathrm{s}}$ for the onset of superconductivity increases. Further the group IV elements $\mathrm{C}, \mathrm{Si}, \mathrm{Ge}, \mathrm{Sn}, \mathrm{Pb}$ are of considerable significance as it nicely shows insulatormetal transition. The last two elements are superconductors with their $T_{\mathrm{c}}$ values decreasing with their atomic radii and similarly the metallization pressures $\left(P_{\mathrm{M}}\right)$ and the superconducting pressures for $\mathrm{Ge}$ and $\mathrm{Si}$ increase with their atomic radii. Further diamond is expected to become metallic at any moment and the trend clearly shows that it should also become superconducting at very high pressures.

It is important to note that there exists a correlation between metallization pressure and atomic radius as was shown by the theoretical calculations of McMahan (1986) for the van der Waals solids also. This correlation relating metallization and superconducting pressures with atomic radii really indicate their connections with the lattice vibrational frequencies or the Debye temperature.

The predicting capability of band structure calculations has been very good with regard to crystal structural transformation and metallization but not with respect to superconducting transition temperature. The calculation of $T_{\mathrm{c}}$ as well as its variation with respect to pressure are somewhat complicated. The factors affecting $T_{\mathrm{c}}$ have been discussed in detail elsewhere (Asokamani et al 1986) and are discussed briefly here which agrees with

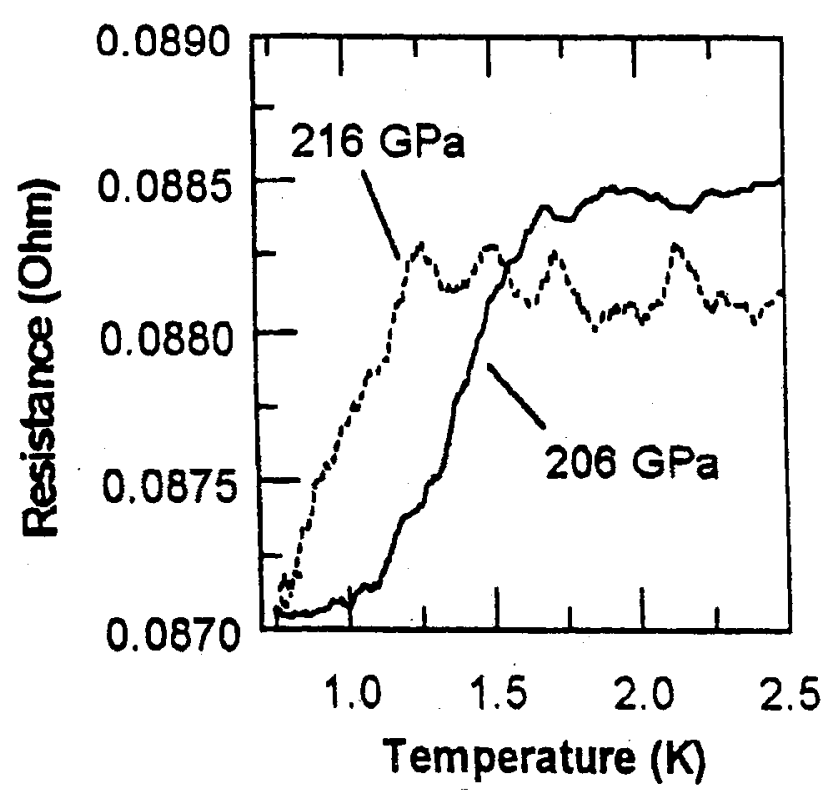

Figure 1. Pressure induced superconductivity in CsI. the experimentalists who have observed a $T_{\mathrm{c}}$ of $2 \mathrm{~K}$ at $1.8 \mathrm{Mbar}$ in CsI and the suggested uncertainties in the calculations.

\section{Phase transformation under pressure}

Before superconductivity occurs the ionic solids should become metals and earlier to this they undergo structural transformations. Most of the alkali iodides usually are transformed from $\mathrm{B} 1$ phase ( $\mathrm{NaCl}$ structure) to $\mathrm{B} 2$ phase $(\mathrm{CsCl}$ structure). The transformation pressures or the corresponding reduced volumes are studied by performing total energy calculations. The total energy of the solid is given by the following expression:

$$
E_{\mathrm{tot}}=T_{\mathrm{kin}}+\sum_{R} U_{R}+\sum_{R} \sum_{R} Z_{R} Z_{R^{\prime}} \sum_{T}\left|R-R^{\prime}-T\right|^{-1},
$$

where the first term is the kinetic energy of the valence electrons which should be expressed as the difference between the total energy and the potential energy of the non-interacting electrons. In atomic sphere approximation, therefore

$$
T_{\mathrm{kin}}=\int_{0}^{E_{\mathrm{k}}} E N(E) \mathrm{d} E-\sum_{R} \int_{0}^{S_{R}} v_{R}(r) n_{R} 4 \pi r^{2} \mathrm{~d} r,
$$

where $N(E)=\Sigma_{R 1} N_{R 1}(E)$ is the sum of the projected density of states, $v_{R}(r)$ is the one electron potential in the sphere at $R$ and $n_{R}(r)$ is the spherically averaged charge density. The second term is the sum of the intra-sphere interaction energy between the electrons themselves and between the electrons and the nucleus in that sphere.

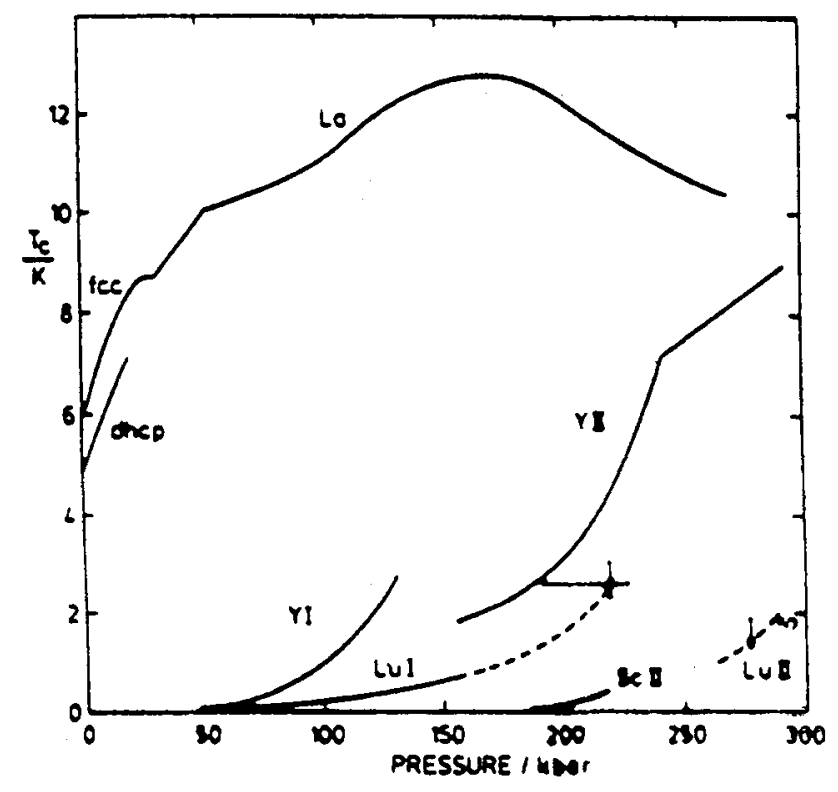

Figure 2. Pressure induced superconductivity in metals. 
The TBLMTO calculations performed by us show the expected transformations as shown below in the case of $\mathrm{RbI}$ and $\mathrm{KI}$ whereas CsI already exists in the B2 phase at ambient pressure. Figure 3 shows the structural transformation in the case of $\mathrm{RbI}$ whereas in table 2, the theoretical reduced volumes are compared with the experimentally quoted values.

There is a continuous $s / p \rightarrow d$ electron transfer in all solids under pressure. The continuous increment in the $d$ electron number is one of the factors, which will promote superconductivity in all solids, and the manner in which $d$ electron number is increasing as a function of pressure is shown below (table 1) in the case of CsI. The other factors which influence superconductivity are discussed below.

\section{Factors affecting $T_{c}$}

First of all the McMillan's formula which has been used to calculate $T_{c}$ has got an exponential dependence on the electron-phonon coupling constant $\lambda$ thus making $T_{\mathrm{c}}$ to be very sensitive to $\lambda$ as shown below:

$$
T_{\mathrm{c}}=\frac{\theta_{\mathrm{D}}}{1.45} \exp \left[-\frac{1.04(1+\lambda)}{\lambda-\mu^{*}(1+0.62 \lambda)}\right],
$$

where

$$
\lambda=\frac{\eta}{\mathrm{M}\left\langle\omega^{2}\right\rangle},
$$

$\eta$ is the electronic parameter which involves the Fermi energy $E_{\mathrm{F}}$, the density of states at $E_{\mathrm{F}}, N\left(E_{\mathrm{F}}\right)$, the wave functions and their derivatives calculated at $E_{\mathrm{F}}$ and corresponding to the Wigner-Seitz sphere radius. These parameters which determine $\eta$ are all calculated syste-

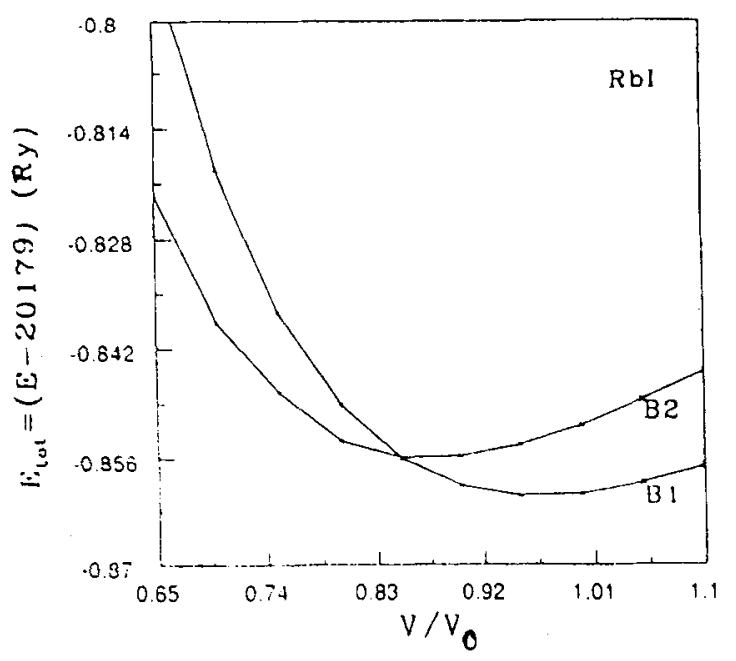

Figure 3. Total energy as a function of volume in $\mathrm{RbI}$ in $\mathrm{Bl}$ and $\mathrm{B} 2$ structures. matically as function of pressure, whereas the variation of phonon frequency with pressure could not be calculated in the same manner. In other words, the phonon part is not calculated in a rigorous manner as that of the electronic part. Soft modes could have occurred during structural transformations and it is a difficult problem to calculate $\left\langle\omega^{2}\right\rangle$ variation with pressure.

However, we, using the Gruniesen constant variation in the case of $\mathrm{La}$, made an attempt in this direction which did improve the calculation of $T_{\mathrm{c}}$ (Asokamani et al 1986). Further the variation of $\theta_{\mathrm{D}}$ as a function of temperature and pressure should be known as it occurs as a pre factor in the McMillan's expression. Because of the above said factors the theoretical calculations can at best give only the trends in the variation of $T_{\mathrm{c}}$ with pressure. Theory has seldom been ahead of the experimental observations. At this juncture it should be said that the theoretical prediction viz CsI will become a superconductor under pressure is indeed a remarkable success of the band theory.

In spite of the above mentioned limitations with regard to the calculation of $T_{c}$, which mainly arise because of the McMillan's equation, still the band theory has been extremely successful in predicting the structural transformations as well as insulator-metal transition in general. In the case of the other alkali iodides the pressure corresponding to structural transitions and insulatormetal transition are given in table 2 and are compared with the experimental results (Mercy Amirthakumari et al 1997).

The systematics discussed so far show that irrespective of the nature of the bonding, the solids undergo the following sequence under pressure: crystal structural transformation, loss of covalency or ionicity, IMT and

Table 1. Density of state at Fermi energy (no. of states/Ry./cell) and the angular momentum decomposed charge distribution (no. of states in electrons/atom) in CsI at different reduced volumes.

\begin{tabular}{lllll}
\hline & & \multicolumn{3}{c}{ No. of states } \\
\cline { 5 - 5 }$V / V_{0}$ & $($ States/Ry. $)$ & $n_{\mathrm{s}}$ & $n_{\mathrm{p}}$ & $n_{\mathrm{d}}$ \\
\hline 0.500 & 0.023 & 1.2 & 5.5 & 0.6 \\
0.450 & 0.107 & 1.19 & 5.4 & 0.7 \\
0.425 & 0.125 & 1.19 & 5.3 & 0.75 \\
0.400 & 0.467 & 1.18 & 5.2 & 0.85 \\
0.325 & 7.210 & 1.2 & 5.0 & 0.95 \\
0.300 & 8.403 & 1.2 & 4.75 & 1.10 \\
\hline
\end{tabular}

Table 2. Metallization volume ratios and the corresponding pressure (in Gpa) given in parenthesis.

\begin{tabular}{lllc}
\hline Compound & Present work & \multicolumn{1}{c}{ Expt. } & Herzfeld \\
\hline $\mathrm{RbI}$ & $0.365(122.3)$ & $0.45(85)$ & 0.363 \\
$\mathrm{Ki}$ & $0.350(154.9)$ & $0.45(115)$ & 0.376 \\
\hline
\end{tabular}


superconductivity. CsI is isoelectronic with that of BaTe and $\mathrm{Xe}$ and the possibility of superconductivity has been already discussed (Subramoniam and Asokamani 1989). Since the ionic solid CsI has become a superconductor, $\mathrm{CsBr}, \mathrm{CsCl}$ and $\mathrm{CsF}$ should also become superconductors under pressure and the pressures required to drive them to go to the superconducting state should be large as the radii of the halide ions are becoming progressively smaller. These studies make us to suggest that all ionic solids should become superconductors at high pressures. Further diamond with is expected to become metallic at any time should also ultimately exhibit superconductivity. The one difference which we have observed with regard to metallization and superconductivity in covalent and ionic solids is that in the former class $P_{\mathrm{M}}=P_{\mathrm{S}}$ whereas $P_{\mathrm{M}} \neq P_{\mathrm{S}}$ in the latter case. We anticipate that the differences between $P_{\mathrm{M}}$ and $P_{\mathrm{S}}$ should become larger with the percentage of ionicity, as in the case of BaTe vs SnTe.

\section{Magnetic transitions under pressure}

The discovery of high $T_{\mathrm{c}}$ superconductors led to the investigations of magnetic insulators using band theory. Similar to the study of equilibrium lattice structures, lattice constants, bulk modulii and their crystallographic modifications under pressure, the total energy calculations performed using spin polarized band structure calculations have been able to give successfully the ground state magnetic structure, magnetic moments and magnetic structural transformations. These studies performed in the case of cubic perovskite oxide systems are given below in table 3 (Pari et al 1995).

\section{Ternary chalcopyrites}

The band structure results obtained for a number of ternary chalcopyrites of the $\mathrm{ABC}_{2}(\mathrm{~A}=\mathrm{Cu}, \mathrm{Ag} ; \mathrm{B}=\mathrm{Ga}$, In; $C=S, S e$ and $T e$ ) type compounds were used to calculate their bulk modulus. Interrelationships connecting cell volume, bulk modulus and Debye temperature were established for as many as 12 systems and they are given below (Asokamani et al 1998).

$$
\begin{aligned}
& B_{0}=-0.075 V_{0}+160.1, \\
& B_{0}=0.107 \theta_{M}-40.059,
\end{aligned}
$$

where $B_{0}, V_{0}$ and $\theta_{\mathrm{M}}$ are respectively the bulk modulus, unit cell volume and melting point. These linear relations are of considerable significance as they are capable of yielding the bulk modulus and melting temperature once the cell volume for any solid crystallizing in this structure is known.

\section{Conclusions}

From our studies we observe that the band gaps are underestimated because of the local density approximation which is well known in the case of band insulators. Further the kind of exchange-correlations usually used in the one-electron band structure calculations-are inadequate and the calculations are incapable of opening up the expected band gaps in the strongly correlated systems. Yet the band structure results quoted in this paper obtained using the LMTO method or the LAPW method are capable of predicting the correct equilibrium structures, the lattice parameters, bulk modulus, crystal structural modifications and metallization pressures. However, the calculations of superconducting transition pressures are somewhat difficult, due to the factors which go into the McMillans formula. The spin polarized band structure calculations give the correct magnetic ground state, magnetic moments and magnetic phase transitions unless the system is strongly correlated.

Our studies on a large number of pressure induced superconductivity make us believe that diamond will be a pressure induced superconductor and experimentalists

Table 3. Variation of insulating $\operatorname{gap}\left(E_{\mathrm{g}}\right)$, magnetic moments and the corresponding phases as function of reduced volume $\left(V / V_{0}\right)$ for the system $\mathrm{LaMO}_{3}(\mathrm{M}=\mathrm{Sc}, \mathrm{Cr}, \mathrm{Fe}$ and $\mathrm{Co})$.

\begin{tabular}{lclcc}
\hline $\mathrm{LaMO}_{3}$ & $\left(V / V_{0}\right)$ & Phase & $\begin{array}{c}E_{\mathrm{g}} \\
(\mathrm{eV})\end{array}$ & $\begin{array}{c}\text { Magnetic } \\
\text { moment of } \\
\mathrm{M} \text { ion }\left(\mu_{\mathrm{B}}\right)\end{array}$ \\
\hline $\mathrm{LaScO}_{3}$ & 0.95 & Insulator & 4.807 & 0.000 \\
& 0.90 & Insulator & 4.941 & 0.000 \\
& 0.85 & Insulator & 5.087 & 0.000 \\
& 0.80 & Insulator & 5.167 & 0.000 \\
& 0.75 & Insulator & 5.093 & 0.000 \\
$\mathrm{LaCrO}_{3}$ & & & & \\
& 0.95 & AFI* & 0.300 & 2.771 \\
& 0.90 & AFI & 0.145 & 2.714 \\
& 0.85 & AFM & 0.000 & 2.650 \\
$\mathrm{LaFeO}_{3}$ & & & & \\
& 1.04 & AFI & 0.541 & 4.144 \\
& 1.02 & AFI & 0.508 & 4.151 \\
& 0.98 & AFI & 0.403 & 4.165 \\
& 0.96 & AFI & 0.359 & 4.172 \\
& 0.94 & AFI & 0.242 & 4.172 \\
& 0.92 & AFI & 0.176 & 4.169 \\
& 0.90 & AFM & 0.000 & 0.872 \\
& 0.88 & AFM & 0.000 & 0.862 \\
$\mathrm{LaCoO}_{3}$ & & & & \\
& 1.10 & Insulator & 0.349 & 0.000 \\
& 1.05 & Insulator & 0.330 & 0.000 \\
& 0.95 & Insulator & 0.514 & 0.000 \\
& 0.90 & Insulator & 0.541 & 0.000 \\
& 0.85 & Insulator & $\mathbf{0 . 5 9 4}$ & 0.000 \\
& 0.60 & Insulator & 0.152 & 0.000 \\
& 0.55 & Metal & 0.000 & 0.000 \\
\hline
\end{tabular}

$\mathrm{AFI}^{*}$, Anti ferromagnetic insulator; and $\mathrm{AFM}^{*}$, anti ferromagnetic metal. 
should search for superconductivity in ionic systems as all ionic systems are anticipated to become superconductors irrespective of the numerical values of $T_{\mathrm{c}}$.

\section{References}

Andersen O K 1975 Phys. Rev. B12 3060

Asokamani R, Suvasini M B, Rajagopalan M and Sundararajan V 1986 Physica B138 94

Asokamani R, Subramoniam G and Mercy Amirthakumari R 1991 Metallization and possible superconductivity in CsI at high pressure, in Proc. of XIII AIRAPT int. conf. on high pressure science and technology (ed.) A K Singh (New Delhi: Oxford and IBH) pp. 434-436

Asokamani R, Ravindran P, Pari G and Mercy Amirthakumari R 1997 Crystal structure, IMT and superconducting transitions in solids under high pressure, in Advances in high pressure research in condensed matter (New Delhi: NISCOM) pp. 278-283

Asokamani R, Mercy Amirthakumari R, Rita R and Ravi C 1998 Phys. Status Solidi (to be published)

Ermets M I, Shimizu K, Kobayashi T C and Amaya K 1998 Science 2811333
McMahan A K 1986 Physica (Amsterdam) 139, B140 31

Mercy Amirthakumari R, Pari G, Rita R and Asokamani R 1997 Phys. Status Solidi B199 157

Mijnarends P E 1973 Physica 63248

Moruzzi V L, Janak J F and Williams A R 1978 Calculated electronic properties of metals (New York: Pergamon Press)

Papaconstantopoulos D A 1986 Handbook of the band structure of the elements (New York: Plenum)

Papaconstantopoulos D A, Boxer L L and Klein B M 1977 Phys. Rev. B15 4221

Pari G, Mathi Jaya S, Subramoniam G and Asokamani R 1995 Phys. Rev. B51 16575

Skriver H L 1984 The LMTO method (Berlin: Springer-Verlag) Skriver H L 1985 Phys. Rev. B31 1909

Slater J C 1937 Phys. Rev. 51846

Subramoniam G and Asokamani R 1989 Possible superconductivity in metallic xenon, in Proc. of XII AIRAPT int. conf. on high pressure science and technology, Paderbon (eds) W B Holzapfel and P G Johannsen (New York: Gordan and Breach) pp. 129-131

Wittig J and Probst C 1978 High-pressure and low-temperature physics (eds) C W Chu and C H Woolam (New York: Plenum) pp. $467-479$ 